\title{
Computer Augmented Modeling of Complexes of L-Phenylalanine and Maleic Acid under Organic Media: Biomimetic Studies
}

\author{
Hadgu Hailekiros Belay \\ Department of Applied Chemistry, Adama Science and Technology University, Adama, Ethiopia \\ Email: hadgu10@gmail.com
}

How to cite this paper: Belay, H.H. (2019) Computer Augmented Modeling of Complexes of L-Phenylalanine and Maleic Acid under Organic Media: Biomimetic Studies. Open Access Library Journal, 6: e5739. https://doi.org/10.4236/oalib.1105739

Received: August 27, 2019

Accepted: September 15, 2019

Published: September 18, 2019

Copyright $\odot 2019$ by author(s) and Open Access Library Inc.

This work is licensed under the Creative Commons Attribution International License (CC BY 4.0).

http://creativecommons.org/licenses/by/4.0/

\section{(c) () Open Access}

\begin{abstract}
Chemical speciation of ternary complexes of $\mathrm{Co}$ (II), $\mathrm{Ni}$ (II) and $\mathrm{Cu}$ (II) ions with L-Phenylalanine and Maleic acid has been studied by Calvin-Wilson technique at different concentration range of $0-50 \% \mathrm{v} / \mathrm{v}$ Organic-water mixture maintaining an ionic strength of $0.16 \mathrm{~mol} \cdot \mathrm{L}^{-1}$ at $298.0 \mathrm{~K}$. Alkali metric titrations were carried out in different relative concentrations (M:L:X = 1:2.5:2.5, 1:2.5:5.0, 1:5.0:2.5) of metal (M) to phenylalanine (L) to maleic acid (X). The different values of stability constants of ternary complexes were calculated, and various models were refined with MINIQUAD75. The best fit chemical models containing MLXH, MLX and $\mathrm{ML}_{2} \mathrm{X}$ for Co (II), Ni (II) and $\mathrm{Cu}$ (II) species were arrived based on stability statistical parameters. The chemical speciation is discussed based on distribution diagrams, drawn using HYSS HYPERQUAD. Effect of errors in concentration of ingredients on stability constants was also studied.
\end{abstract}

\section{Subject Areas \\ Analytical Chemistry}

\section{Keywords}

Chemical Speciation, Ternary Complexes, Phenylalanine, Maleic Acid, Urea, MINIQUAD75

\section{Introduction}

L-phenylalanine (F) is an essential non-polar $\alpha$-amino acid because of the hydrophobic nature of the benzyl side chain. It is found naturally in the breast milk of mammals. It is used in the manufacture of food and drink products and sold 
as a nutritional supplement for its reputed analgesic and antidepressant effects. It is a direct precursor to the neuro modulator phenyl ethylamine, F commonly used dietary supplement. It is an antagonist at higher doses; this may play a role in its analgesic and antidepressant properties. It is the starting compound used in the flavonoid biosynthesis. It is converted to cinnamic acid by the action of enzyme phenylalanine ammonialyase [1].

Maleic acid (Ma) is an organic compound. It is an unsaturated dicarboxylic acid, a molecule with two carboxyl groups. It is the cis isomer of butene dioic acid, whereas fumaric acid is the trans-isomer. It is mainly used as a precursor to fumaric acid, and relative to its parent maleic anhydride. It is more soluble in water. Ma and fumaric acid do not spontaneously interconvert because rotation around a carbon-carbon double bond is not energetically favorable. However, conversion of the cis isomer into the trans-isomer is possible by photolysis in the presence of a small amount of bromine [2].

Urea acts as a denaturant of macromolecules but this action is reversible. The denaturing effect of urea on proteins can occur in two ways: 1) Direct interaction of urea molecules with amide and peptide groups, to destroy intermolecular hydrogen bonds and hydrophobic interaction [3] that are involved in the maintenance of ternary structures, and 2) Urea molecules exert their influence through changes in water structure. [4] The increased solubilities of amino acids [5] and hydrocarbons [6] in presence of urea supports the former mechanism. The negative heat capacities of transfer of certain amino acids [7] from water to aqueous urea solution and the increments in the critical micellar concentration [8] of certain surfactants suggest that urea may reduce the co-operative structure of water.

Urea-water mixture was selected as media to maintain the dielectric properties of the medium in comparable levels to those of the physiological fluids since the polarity at the active site cavities should generally be applicable when it is possible to compare ligand binding to the metal ion as in protein residues and solvent environment [9]. This study is undergoing to investigate chemical speciation of ternary complexes of $\mathrm{Co}$ (II), $\mathrm{Ni}$ (II) and Cu (II) with L-phenylalanine and maleic acid in aqueous and Urea-water mixtures.

\section{Experimental}

All the chemicals used were of analytical reagents grade. L-phenylalanine, maleic acid, $\mathrm{HCl}$, methanol, acetone or diethyl ether, triple distilled water, $\mathrm{NaOH}$ pellet, hexamethylenetetramine powder, oxalic acid, potassium hydrogen phthalate, borax, Urea, EDTA, acetic acid, 2-amono ethanol, $\mathrm{ZnSO}_{4}, \mathrm{CoCl}_{2}, \mathrm{CuCl}_{2}, \mathrm{NiCl}_{2}$, $\mathrm{NaCl}$, Eriochrome-black. $\mathrm{T}$ as indicators, xylenol orange, murexide and sulphone black-F as indicator.

\subsection{Experimental Procedure}

\section{Preparation of Solutions}

All solutions were prepared in boiled out triple distilled water. 


\section{1) L-phenylalanine and Maleic acid Solutions}

0.05 moldm $^{-3}$ aqueous solution of L-phenylalanine (GR grade, E-Merck, Germany) and maleic acid (GR grade, E-Merck, Germany) were prepared by dissolving samples in water. To increase the solubility of ligands, 0.05 moldm $^{-3}$ hydrochloric acid concentration was maintained in the solutions. The probable errors that may creep into the concentrations of the stock solutions of the ligands were determined by the computer program COSWT [10]. The pessimistic errors in the preparation of the ligand solutions by weight method did not exceed $0.1 \%$.

\section{2) EDTA Solution}

Disodium salt of EDTA was purified by precipitation from aqueous solution using methanol. The precipitate was washed with acetone, diethyl ether and finally air dried. An $0.1 \mathrm{moldm}^{-3}$ solution was prepared and standardized complex metrically with a 0.1 moldm $^{-3}$ standard Zn (II) solution using Eriochrome Black-T as indicator.

\section{3) Metal ion Solutions}

0.1 moldm $^{-3}$ aqueous solutions of cobalt (II), nickel (II) and copper (II) chlorides were prepared by dissolving GR grade (E-Merck, India) salts in triple distilled water. The stock solutions were rendered slightly acidic to repress hydrolysis of the metal ions. The concentrations of the metal ions were determined complex metrically by titrating against a standard solution of EDTA using the xylenol orange, murexide and fast sulphon black-F as indicators and hexamethylenetetramine powder as buffer for cobalt to maintain the $\mathrm{pH}$ at $5-6$. The free hydrogen ion concentration in the stock solution was determined by Gran plot method [11].

\section{4) Sodium Hydroxide}

A stock solution of $1 \mathrm{moldm}^{-3}$ sodium hydroxide was prepared by dissolving GR grade (E Merck, India) sodium hydroxide pellets in triple distilled water. The solution was further diluted to the required concentration. The strength of the alkali was determined by titrating it against a standard solution of oxalic acid and potassium hydrogen phthalate, while the molarity of hydrochloric acid was determined with sodium hydroxide. So as to assess the errors that might have crept into the determination of the concentrations, the data were subjected to analysis of variance of one way classification (ANOVA) using the computer program COST (concentration of solution by titration). The strength of alkali was determined using the Gran plot method. The errors in the concentration of ligand, metal ions and alkali were subjected to analysis of variance ANOVA [12].

\section{5) Hydrochloric Acid}

The concentration of stock hydrochloric acid (GR grade, Merck, India) solution was calculated from its specifications (specific gravity, purity and molecular weight) and diluted to the required concentration by dissolving in triple distilled water. Its strength was determined by titrating with standard sodium hydroxide solution. 


\subsection{Solvent}

Analargrade $(\mathrm{BDH})$ urea was recrystallized twice from triply distilled water and was dried at $60^{\circ} \mathrm{C}$ for $2 \mathrm{~h}$. A stock solution of urea was prepared by dissolving and appropriate quantity of the purified sample in water and stored in frozen state. The solution was not allowed to stand at room temperature continuously for more than $24 \mathrm{~h}$.

GR grade N, Urea (Finar, India) of $99.5 \%$ purity was used as a solvent.

\subsection{Titration Procedure}

The $\mathrm{pH}$ measurements of the proton-ligand systems were carried out in aqueous media containing varying compositions of organic solvent (Urea) in the range of $0 \%-50 \% \mathrm{v} / \mathrm{v}$ maintaining an ionic strength of 0.16 moldm $^{-3}$ with sodium chloride at $298 \mathrm{~K}$ using a digital $\mathrm{pH}$ meter ELICO-LI120 type (readability 0.01). Potassium hydrogen phthalate $\left(0.05\right.$ moldm$\left.^{-3}\right)$ and borax $\left(0.01 \mathrm{moldm}^{-3}\right)$ solutions were used to calibrate the $\mathrm{pH}$ meter. In each titration, the titrand consisted of approximately $1 \mathrm{mmol}$ of nitric acid. The amounts of the L-phenylalanine, maleic acid (ligands) in the titrand are in the range of $0.25-0.50 \mathrm{mmol}$. The glass electrode was equilibrated in a well stirred Urea-water mixture containing inert electrolyte for several days. At regular intervals, the strong acid was titrated against alkali to check the complete equilibration of the glass electrode. In these titrations, the titrand consisted of mineral acid and ligand, in a total volume of $50 \mathrm{~mL}$. Titrations were performed by adding each time $0.1 \mathrm{~cm}^{3}$ portions of 0.4 moldm ${ }^{-3}$ sodium hydroxide to the titrand. The $\mathrm{pH}$ meter reading was recorded only after a constant value was displayed. Typical duplicate titrations showed that equilibration was fast and titration data did not differ by more than 0.02 units [13].

\subsection{Metal-Ligand Equilibria}

In each of the titrations, the titrand consisted of approximately $1 \mathrm{mmol}$ hydrochloric acid in a total volume of $50 \mathrm{~mL}$ and the ionic strength was adjusted to $0.16 \mathrm{~mol} \cdot \mathrm{L}^{-1}$ with sodium chloride. Solutions containing metal ions and ligands (metal to ligand ratios being in the range of 1:2.5:2.5, 1:2.5:5.0, 1:5.0:2.5 were titrated with $0.4 \mathrm{~mol} \cdot \mathrm{L}^{-1}$ sodium hydroxide.

\subsection{Modeling Strategy}

The approximate protonation constants of L-phenylalanine and maleic acid were calculated with the computer program SCPHD [14]. The best fit chemical model for each system investigated was arrived at using non-linear least squares computer program, MINIQUAD75, which exploits the advantage of constrained least-squares method in the initial refinement and reliable convergence of Marquardt algorithm. The variation of stepwise protonation constants $(\log K)$ with the mole fraction of the medium was analyzed on electrostatic grounds for the solute-solute and solute-solvent interactions [15]. 


\section{Result and Discussion}

The results of the final best-fit models that reveal the stoichiometry of the complex species and their overall formation constants along with some of the associated statistical parameters are given in Table 1 . Very low-standard deviation in overall stability constants $(\log \beta)$ signifies the precision of these data. The small values of Ucorr (sum of squares of deviations in concentrations of ingredients at all experimental points) corrected for degrees of freedom, small values of mean, standard deviation and mean deviation for the systems studied are validated by the residual analysis [16] [17].

The concentrations of the metal, the ligands and the hydrogen ion at all experimental points corrected for degrees of freedom indicate that the models represent the experimental data. Small values of mean, standard deviation and mean deviation for the systems corroborate that the residuals are around a zero

Table 1. Parameters of best fit chemical model of F-M (II)-MA complexes in Urea-water mixtures. Temperature $=298 \mathrm{~K}$, Ionic strength $=0.16$ moldm $^{-3}$.

\begin{tabular}{|c|c|c|c|c|c|c|c|c|c|c|}
\hline \multirow{2}{*}{$\begin{array}{l}\text { Urea } \\
\% \mathrm{v} / \mathrm{v}\end{array}$} & \multicolumn{3}{|c|}{$\log \beta_{\mathrm{mlh}}(\mathrm{SD})$} & \multirow{2}{*}{-pH-Range } & \multirow{2}{*}{ NP } & \multirow{2}{*}{$\begin{array}{l}\mathrm{U}_{\text {corr }} \\
{ }^{\star} 10^{8}\end{array}$} & \multirow{2}{*}{$x^{2}$} & \multirow{2}{*}{ Skew-ness } & \multirow{2}{*}{ Kurt-osis } & \multirow{2}{*}{ R-factor } \\
\hline & MLXH & MLX & $\mathrm{ML}_{2} \mathrm{X}$ & & & & & & & \\
\hline \multicolumn{11}{|c|}{$\mathrm{Co}(\mathrm{II})$} \\
\hline 0.0 & $19.62(7)$ & $12.90(0)$ & $17.09(9)$ & $1.5-8.9$ & 44 & 5.37 & 4.90 & 0.06 & 3.33 & 0.0081 \\
\hline 10 & $20.19(6)$ & $13.00(5)$ & $17.30(1)$ & $1.5-8.9$ & 67 & 4.15 & 3.44 & 0.01 & 1.17 & 0.0022 \\
\hline 20 & $21.10(9)$ & $14.69(9)$ & $18.45(0)$ & $1.5-8.6$ & 85 & 8.00 & 2.22 & -0.01 & 4.16 & 0.0006 \\
\hline 30 & $21.19(3)$ & $15.18(0)$ & $19.40(0)$ & $1.5-8.6$ & 88 & 5.14 & 4.55 & 0.17 & 7.00 & 0.0040 \\
\hline 40 & $22.17(5)$ & $14.95(7)$ & $19.017(3)$ & $1.5-8.8$ & 66 & 1.05 & 9.11 & -0.15 & 3.01 & 0.0034 \\
\hline 50 & $22.19(3)$ & $15.66(9)$ & $20.20(2)$ & $1.5-8.7$ & 99 & 1.07 & 6.23 & -0.12 & 1.03 & 0.0035 \\
\hline \multicolumn{11}{|c|}{$\mathrm{Ni}(\mathrm{II})$} \\
\hline 0.0 & $19.16(1)$ & $12.50(4)$ & $17.70(0)$ & $1.5-7.6$ & 66 & 2.53 & 0.93 & -0.09 & 4.10 & 0.0010 \\
\hline 10 & $20.37(0)$ & $13.83(5)$ & $19.24(12)$ & $1.5-7.6$ & 74 & 1.33 & 5.71 & -0.07 & 3.13 & 0.0018 \\
\hline 20 & $21.00(6)$ & $14.90(9)$ & 19.65(9) & $1.5-7.6$ & 88 & 5.22 & 4.15 & -0.80 & 3.09 & 0.0024 \\
\hline 30 & $20.17(3)$ & $13.85(7)$ & $19.80(0)$ & $1.8-8.6$ & 90 & 3.31 & 3.12 & 0.60 & 3.06 & 0.0030 \\
\hline 40 & $21.10(9)$ & $14.40(6)$ & $19.66(7)$ & $1.6-8.6$ & 56 & 1.30 & 3.86 & -0.19 & 1.00 & 0.0061 \\
\hline 50 & $19.06(8)$ & $12.72(0)$ & $18.45(9)$ & $1.6-8.6$ & 77 & 2.19 & 1.55 & -1.00 & 3.51 & 0.0050 \\
\hline \multicolumn{11}{|c|}{$\mathrm{Cu}(\mathrm{II})$} \\
\hline 0.0 & $19.70(1)$ & $14.89(5)$ & $21.90(5)$ & $1.7-6.4$ & 99 & 3.22 & 1.20 & 0.09 & 2.60 & 0.0008 \\
\hline 10 & $20.19(8)$ & $14.97(6)$ & $21.50(4)$ & $1.7-6.4$ & 45 & 3.70 & 2.30 & -0.01 & 2.80 & 0.0023 \\
\hline 20 & $21.30(0)$ & $16.36(4)$ & $23.67(3)$ & $1.7-6.4$ & 90 & 629 & 3.17 & -0.10 & 3.05 & 0.0008 \\
\hline 30 & $21.00(17)$ & $16.15(8)$ & $23.30(1)$ & $1.8-6.4$ & 34 & 4.22 & 9.14 & 0.32 & 2.31 & 0.0090 \\
\hline 40 & $22.51(6)$ & $17.29(8)$ & $23.74(0)$ & $2.1-6.4$ & 67 & 3.12 & 7.00 & -0.36 & 3.10 & 0.0099 \\
\hline 50 & $21.17(9)$ & $16.91(7)$ & $24.80(9)$ & $2.1-6.4$ & 88 & 6.10 & 5.27 & 0.09 & 3.45 & 0.0010 \\
\hline
\end{tabular}

$\mathrm{Ucorr}=\mathrm{U} /(\mathrm{NP}-\mathrm{m}) \mathrm{X} 108$, where $\mathrm{m}=$ number of species; $\mathrm{NP}=$ Number of experimental points; $\mathrm{SD}=$ standard deviation. 
mean with little dispersion. For an ideal normal distribution, the values of kurtosis and skewness should be three and zero, respectively. The kurtosis values in the present study indicate that most of the residuals are very nearer to leptokurtic and a few form platykurtic pattern whose values are less than 3 . The values of skewness recorded in Table 1 are between -1.00 and 0.60 in Urea-water media show that the residuals form a part of normal distribution and hence a least squares method can be applied to the present data. The sufficiency of the model is further evident from the low crystallographic $\mathrm{R}$ factor values, which indicate the need for inclusion of additional species in the model. $\mathrm{X}^{2}$ is a special case of gama distribution which measures the probability of residuals forming a part of standard normal distribution [18]. All the metal complexes of the form $\mathrm{MLX}_{2} \mathrm{H}_{\mathrm{h}}$ is rejected by the program MINIQUAD75. This may be because of the instability of these complexes due to the inability of the metal ions to accommodate three bulky ligands. The reasons for the existence of different species are ascribed under the head distribution diagrams.

$$
\chi^{2} \text { test }
$$

$\chi^{2}$ is a special case of gamma distribution whose probability density function is an unsymmetrical function. This distribution measures the probability of residuals forming a part of standard normal distribution with zero mean and unit standard deviation.

\section{Crystallographic R-test}

In crystallography, the R-factor (sometimes called residual factor or reliability factor) is a measure of the agreement between the crystallographic model and the experimental X-ray diffraction data. The minimum possible value is zero, indicating perfect agreement between experimental observations and the structure factors predicted from the model. There is no theoretical maximum, but in practice, values are considerably less than one even for poor models, provided the model includes a suitable scale factor. Hamilton's R factor ratio test is applied in complex equilibria to decide whether inclusion of more species in the model is necessary or not. In $\mathrm{pH}$ metric method the readability of $\mathrm{pH}$ meter is taken as the Rlimit, which represents the upper boundary of $\mathrm{R}$ beyond which the model bears no significance. When different values are obtained for models containing different numbers of species, models whose values are greater than $\mathrm{R}$-table are rejected. The low crystallographic $\mathrm{R}$ values given in Table 1 indicate the sufficiency of the model [19].

\section{Skewness}

Skewness is a measure of the symmetry in a distribution. Conceptually, skewness describes which side of a distribution has a longer tail. If the long tail is on the right, then the skewness is rightward or positive; if the long tail is on the left, then the skewness is leftward or negative. In otherwise, if the skewness is greater than zero, the peak of the error distribution curve is to the left of mean and the peak is to the right of the mean if skewness is less than zero. The values of skewness recorded in Table 1 are between -0.15 and 0.17 forCo (II), -1.00 and 0.60 for $\mathrm{Ni}$ (II) $\&-0.36$ and 0.32 for $\mathrm{Cu}$ (II) in Urea-water medium. This data evince 
that the residuals form a part of normal distribution; hence, least-squares method can be applied to the present data [20].

\section{Kurtosis}

Kurtosis is a measure of whether the data are heavy-tailed or light-tailed relative to a normal distribution. For an ideal normal distribution kurtosis value should be three (mesokurtic). If the calculated kurtosis is less than three, the peak of the error distribution curve is flat (platykurtic) and if the kurtosis is greater than three, the distribution shall have sharp peak (leptokurtic). The kurtosis values in the present study indicate that the residuals form leptokurtic pattern.

\subsection{Effect of Systematic Errors}

In order to rely upon the best-fit model for critical evaluation and application under varied experimental conditions with different accuracies of data acquisition, an investigation was undertaken by introducing pessimistic errors in the influential parameters. The results of effect of pessimistic errors in the concentrations of alkali, mineral acid, ligands and metal are given in Table 2 which

Table 2. Effect of errors in influential parameters on the stability constants of ternary complexes of $\mathrm{Ni}$ (II) with $\mathrm{F}$ and MA in $10 \% \mathrm{v} / \mathrm{v}$ Urea-water mixture.

\begin{tabular}{|c|c|c|c|c|}
\hline \multirow{2}{*}{ Ingredient } & \multirow{2}{*}{$\%$ Error } & \multicolumn{3}{|c|}{$\log \beta(\mathrm{SD})$} \\
\hline & & MLXH & MLX & $\mathrm{ML}_{2} \mathrm{X}$ \\
\hline \multirow{5}{*}{ Alkali } & 0 & $20.37(0)$ & $13.83(5)$ & $19.24(12)$ \\
\hline & -5 & $20.80(29)$ & $12.44(69)$ & Rejected \\
\hline & -2 & Rejected & $13.22(55)$ & $18.67(50)$ \\
\hline & 2 & Rejected & Rejected & $20.16(59)$ \\
\hline & 5 & Rejected & Rejected & Rejected \\
\hline \multirow{4}{*}{ Acid } & -5 & Rejected & $16.39(53)$ & Rejected \\
\hline & -2 & $21.3440)$ & $14.55(80)$ & $19.83(59)$ \\
\hline & 2 & $21.84(90)$ & Rejected & $18.90(59)$ \\
\hline & 5 & Rejected & $12.28(60)$ & Rejected \\
\hline \multirow{4}{*}{$\mathrm{F}$} & -5 & $20.07(55)$ & $13.50(45)$ & $19.46(49)$ \\
\hline & -2 & $20.07(77)$ & $13.64(66)$ & $19.45(68)$ \\
\hline & 2 & $20.14(47)$ & $13.68(65)$ & $19.44(36)$ \\
\hline & 5 & $20.43(26)$ & $14.98(29)$ & $19.52(38)$ \\
\hline \multirow{4}{*}{ MA } & -5 & $20.17(33)$ & $14.06(33)$ & $19.37(39)$ \\
\hline & -2 & $20.13(17)$ & $13.81(33)$ & $18.82(39)$ \\
\hline & 2 & $21.07(27)$ & $13.48(39)$ & $19.08(49)$ \\
\hline & 5 & $20.05(18)$ & $13.06(39)$ & $19.37(52)$ \\
\hline \multirow{4}{*}{ Metal } & -5 & $20.17(16)$ & $13.82(13)$ & $19.72(23)$ \\
\hline & -2 & $20.13(17)$ & $13.74(29)$ & $19.53(39)$ \\
\hline & 2 & $20.08(17)$ & $13.61(35)$ & $19.36(39)$ \\
\hline & 5 & $20.07(17)$ & $13.49(29)$ & $19.18(49)$ \\
\hline
\end{tabular}


emphasize that the errors in alkali and acid affect stability constant more than those in the ligands and metal. Some species are even rejected when errors are introduced in the concentrations. This indicates the appropriateness of the experimental conditions and accuracy of the concentrations.

In order to rely upon the best chemical model for critical evaluation and application under varied experimental conditions with different accuracies of data acquisition, an investigation was made by introducing pessimistic errors in the influential parameters like concentrations of alkali, mineral acid, ligand and metal. The order of the ingredients that influence the magnitudes of stability constants due to incorporation of errors is alkali $>$ acid $>$ MA $>$ Phe $>$ metal (Table 2). Some of the species refined in the absence of errors were even rejected when errors were introduced in the concentrations. One or more of MLXH, MLX and $\mathrm{ML}_{2} \mathrm{X}$ species were rejected depending upon the magnitude of error as given in Table 2. The rejection of species and increased standard deviations in the stability constants on introduction of errors confirm the appropriateness or correctness of the experimental conditions (concentrations of ingredients) and the choice of the best fit models.

\subsection{Stability of Ternary Complexes}

The formation of mononuclear unprotonated binary and ternary complexes from a mixture of metal ion $(\mathrm{M})$ and primary $(\mathrm{L})$ and secondary $(\mathrm{X})$ ligands can be shown as the equilibria given in (1).

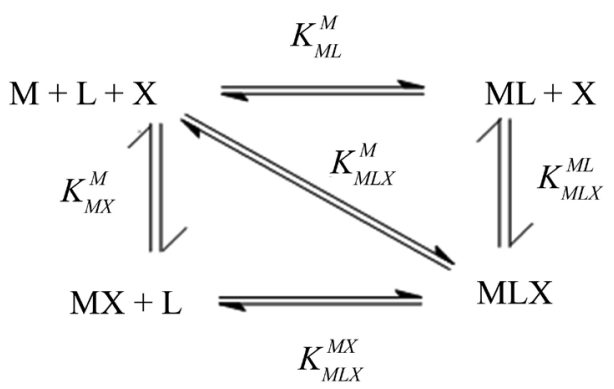

\section{Reasons for extra stability of ternary complexes}

The change in the stability of the ternary complexes as compared to their binary analogues was quantified [21] [22] [23] [24] based on the disproportionation constant $(\log X)$ given by Equation (2) corresponding to the equilibrium.

$$
\begin{gathered}
\mathrm{ML}_{2}+\mathrm{MX}_{2} \rightleftharpoons 2 \mathrm{MLX} \\
\log X=2 \log K_{M L X}^{M}-\log K_{M L_{2}}^{M}-\log K_{M X_{2}}^{M}
\end{gathered}
$$

Under these equilibrium conditions one can expect 50\% ternary complex and $25 \%$ each of the binary complexes to be formed and the value of $\log X$ was reported [25] to be 0.6. A value greater than this accounts for the extra stability of MLX. Another approach [26] to quantify the stability of ternary complexes was based on the difference in stability $(\Delta \log K)$ for the reactions $M L$ with $\mathrm{X}$ and $\mathrm{M}_{(\mathrm{aq})}$ with $\mathrm{L}$ and $\mathrm{X}$, where $\mathrm{L}$ is primary ligand and $\mathrm{X}$ is the secondary ligand. It is compared with that calculated purely on statistical grounds. Equation 5.3 can be 
formulated based on the properties of the cyclic systems reported earlier [27] from which it is clear that both the ligands in the ternary complex influence mutually to the same extent.

$$
\Delta \log K=\log K_{M L X}^{M}-\log K_{M L}^{M}-\log K_{M X}^{M}
$$

The electrostatic theory of binary complex formation and statistical arguments suggest the additional coordination positions of given multivalent hydrated metal ion available for the first ligand than for the second. Hence, the usual order of stability $K_{M L}^{M}>K_{M L X}^{M L}$ applies. This suggests that $\Delta \log K$ should be negative, although several exceptions [28] have been found. The statistical values of $\Delta \log K$ for bidentate $\mathrm{L}$ and $\mathrm{X}$ are $-0.4,-0.6$ and between -0.9 and -0.3 for octahedral, square planar and distorted octahedral complexes, respectively. Negative values of $\Delta \log K$ can be understood as the secondary ligand forms a more stable complex with hydrated metal ion than with ML. Whenever the experimental values of $\Delta \log K$ exceed the statistical values, it can be inferred that the ternary complex is formed as a result of interaction of ML with X or MX with $\mathrm{L}$. $\Delta \log K$ values of ternary complexes containing bipyridyl as the primary ligand are positive [29] for O-donors (malonic acid, pyrocatechol etc.), negative for $\mathrm{N}$-donors (ethylene diamine) and intermediate or negative for amino acids with both $\mathrm{N}$ and $\mathrm{O}$ co-ordination sites. However, a very high negative value $(-2.3)$ for $\mathrm{Cu}$ (en) (iminodiacetic acid) and a positive value (0.82) for $\mathrm{Cu}(\mathrm{o}-\mathrm{F})$-(6, 7-dihydroxynaphthaline-2 sulphonate) was also observed [30].

\section{Effect of Solvent}

The variations of stability constants as a function of dielectric constant of the medium are shown in Figures 1(A)-(C). The variation of overall stabilities constant values or change in free energy with co-solvent content depends upon two factors, viz., electrostatic and non-electrostatic contribution to the free energy change. Hence, the $\log \beta$ values should vary linearly as a function of dielectric constant of the medium, indicates that electrostatic forces and decreasing dielectric constant of the medium [31] [32] are dominating the equilibrium process under present experimental condition. The increase in entropy which is generally observed when the association process is due to electrostatic forces, is caused

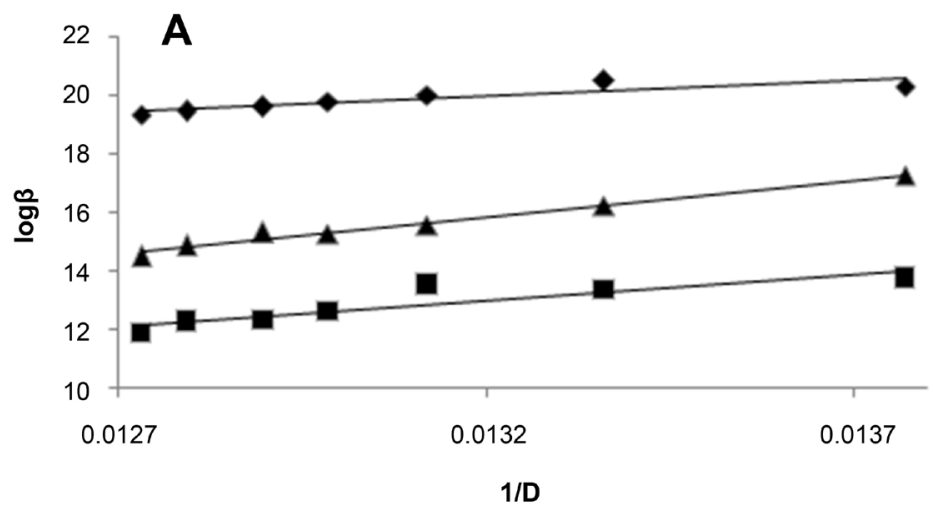




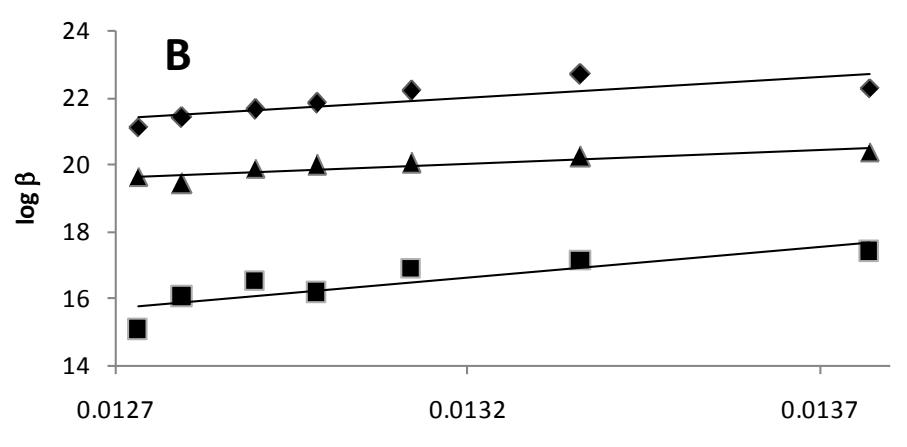

$1 / \mathrm{D}$

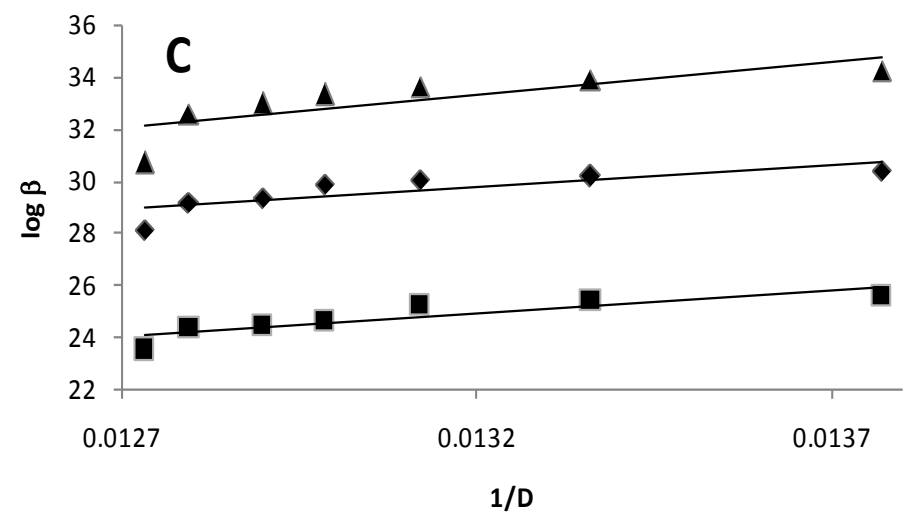

Figure 1. Variation of stability constants of ternary complexes with mole fraction of Urea. (A) Co (II), B) Ni (II) and C) Cu (II) (•) $\log \beta_{\mathrm{MLXH}},(\bullet) \log \beta_{\mathrm{MLX}},(\boldsymbol{\Delta}) \log \beta_{\mathrm{ML} 2 \mathrm{X}}$.

by the negative temperature coefficient of the dielectric constant of the solvent. The greater the electrostriction around the species associating on account of purely electrostatic forces, the more exothermic the reaction will be. The structural changes exerted by a given metal cation on its solvation shell depend not only on its charge and radius, but also on its individuality.

\section{Distribution Diagrams}

Distribution diagrams were drawn using the formation constants of the best fit model and are shown below.

\subsection{Organic Water-Media}

A perusal of the distribution diagrams (Figures 2(A)-(F)) reveals that the concentrations of binary species are less compared to ternary species which indicates the existence of more stable ternary complexes. The ternary species exist in the $\mathrm{pH}$ range $1.5-8.9$ for all the metal ions (Co (II), Ni (II) and $\mathrm{Cu}$ (II). The formation of the complex species can be represented by the following equilibria.

$$
\begin{aligned}
\mathrm{M}(\mathrm{II})+\mathrm{LH}_{2}^{+}+\mathrm{XH}_{2} & \rightleftharpoons \mathrm{MLXH}+3 \mathrm{H}^{+} \\
\mathrm{MLH}^{2+}+\mathrm{XH}_{2} & \rightleftharpoons \mathrm{MLXH}+2 \mathrm{H}^{+} \\
\mathrm{MXH}^{+}+\mathrm{LH}_{2}^{+} & \rightleftharpoons \mathrm{MLXH}+2 \mathrm{H}^{+} \\
\mathrm{MLXH} & \rightleftharpoons \mathrm{MLX}^{-}+\mathrm{H}^{+}
\end{aligned}
$$



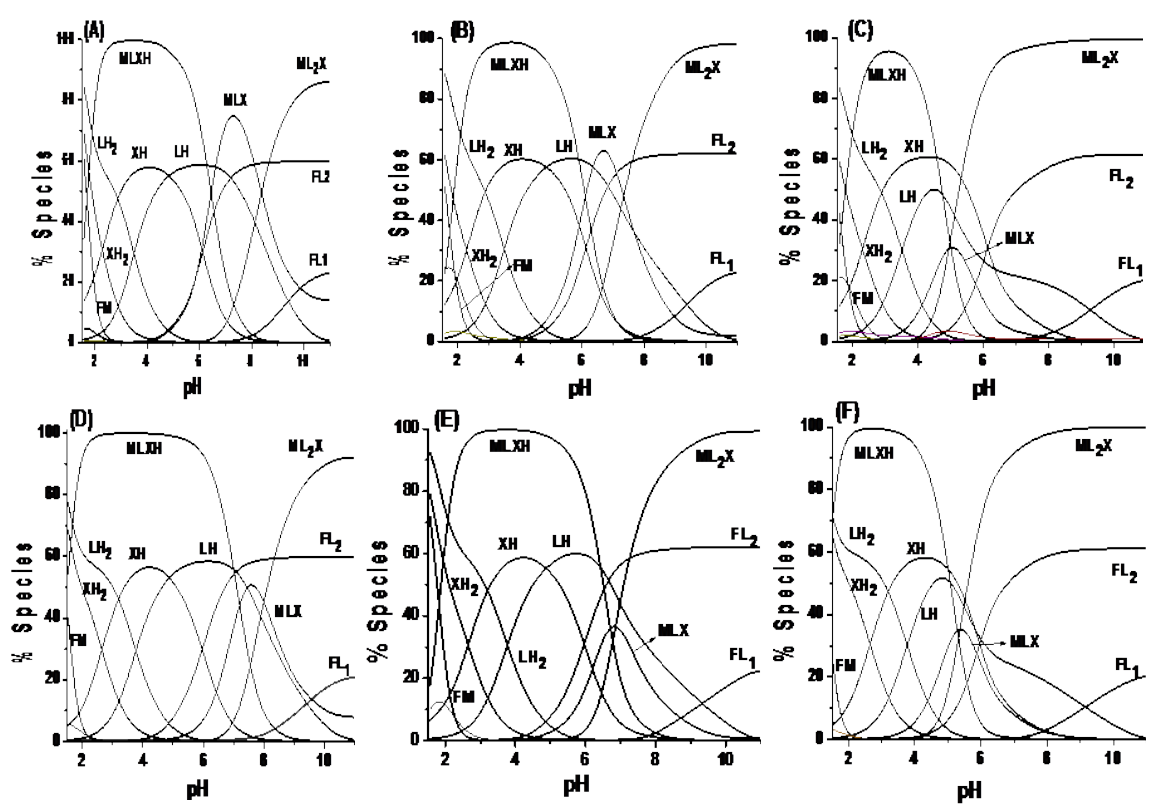

Figure 2. Distribution diagrams for ternary complexes of F and MA with (A) Co (II), (B) $\mathrm{Ni}$ (II) and (C) $\mathrm{Cu}$ (II) in $1.0 \% \mathrm{w} / \mathrm{v}$ and (D) Co (II), (E) $\mathrm{Ni}$ (II) and (F) $\mathrm{Cu}$ (II) in $2.0 \%$ w/v Urea-water mixtures.

$$
\begin{aligned}
\mathrm{ML}_{2} \mathrm{H}^{+}+\mathrm{XH}_{2} & \rightleftharpoons \mathrm{ML}_{2} \mathrm{X}^{2-}+3 \mathrm{H}^{+} \\
\mathrm{ML}_{2}+\mathrm{XH}^{-} & \rightleftharpoons \mathrm{ML}_{2} \mathrm{X}^{2-}+\mathrm{H}^{+} \\
\mathrm{MLX}^{-}+\mathrm{LH} & \rightleftharpoons \mathrm{ML}_{2} \mathrm{X}^{2-}+\mathrm{H}^{+}
\end{aligned}
$$

In the $\mathrm{pH}$ range $2.1-11.4$ and $1.6-10.2, \mathrm{~F}$ and MA exist as $\mathrm{LH}_{2}^{+}, \mathrm{LH}, \mathrm{L}^{-}$and $\mathrm{XH}_{2}, \mathrm{XH}^{-}, \mathrm{X}^{2}$ respectively. These protonated ligands interact with the metal ion to form MLXH (Equilibrium 4) which may successfully deprotonated to form $\mathrm{MLX}^{-}$(Equilibria 7). Formation of MLXH species can be explained based on the protonated ligands interact with the metal ion to form MLXH (Equilibrium 4) and also due to interaction of binary species with ligand species (Equilibrium 5 and 6). $\mathrm{MLXH}_{2}$ species has not been detected probably because it is less stable or quickly deprotonated to MLXH at lower $\mathrm{pH}$ whereas for the formation of $\mathrm{MLX}^{-}$, Equilibria 7 is relevant because the $\mathrm{pH}$ at which the concentration of MLXH decreases, $\mathrm{MLX}^{-}$increases in the $\mathrm{pH}$ range of 2.0 - 5.1. $\mathrm{ML}_{2} \mathrm{X}^{2-}$ is formed by the interaction of metal ion with to $\mathrm{XH}_{2}, \mathrm{LH}$ species and one $\mathrm{XH}^{-}$species (Equilibrium 8, 9 and 10). The existence of $\mathrm{ML}_{2} \mathrm{X}$ and the absence of $\mathrm{MLX}_{2}$ may be due to the higher affinity of $\mathrm{LH}$ than $\mathrm{XH}$ towards the metal ion.

\subsection{Structures}

In aqueous solutions, $\mathrm{Co}$ (II), $\mathrm{Ni}$ (II) and $\mathrm{Cu}$ (II) are coordinated by six water molecules. Amino nitrogen can associate with a proton at physiological $\mathrm{pH}$. There is often significant competition between proton and metal ion for this donor site, resulting in the formation of protonated species. Depending upon the nature of the ligands and metal ions and based on the basic chemical knowledge, 
structures of the complexes are shown in Figure 3. The $\mathrm{Cu}$ (II) ion forms distorted octahedral or square planar complexes due to Jahn-Teller effect.

\section{Conclusions}

1) In this study, species detected as $M L X H, M L X$ and $M_{2} X$ for Co (II), Ni (II), and $\mathrm{Cu}$ (II) where $\mathrm{L}=\mathrm{F}$ and $\mathrm{X}=\mathrm{Ma}$. The active forms of these ligands are: $\mathrm{LH}_{2}^{+}, \mathrm{LH}$ and $\mathrm{L}^{-}$for $\mathrm{F}$ and $\mathrm{XH}_{2}, \mathrm{XH}^{-}, \mathrm{X}^{2-}$ for $\mathrm{Ma}$.

2) The $\Delta \log K$ values indicate that the ternary species have extra stability compared to their binary species, may be due to the interactions outside the coordination sphere, such as the formation of hydrogen bonds between the coordinate ligands, Charge neutralization, chelate effect, stacking interactions and electro static interaction between non-coordinated charge groups of the ligand.

3) The linear increase in conditional stabilities of ternary complexes with decreasing dielectric constant is due to the dominance of electrostatic force.

4) Effect of systematic errors in the influential parameters shows that the errors in the concentration of alkali and mineral acid were found to affect more than that of the ligand did.

5) The study gives an insight in to the metal availability/metal transport in bio fluids and toxicity of these metals. The ternary complexes and more amenable for "metal transport" because of their extra stability and the binary complexes make the "metal available" in biological system due to their decrease conditional stability.

6) The pre dominant species detected at physiological PH is MLX for Co (II), $\mathrm{Ni}$ (II) and $\mathrm{Cu}$ (II) where $\mathrm{L}=\mathrm{F}$ and $\mathrm{X}=\mathrm{Ma}$.

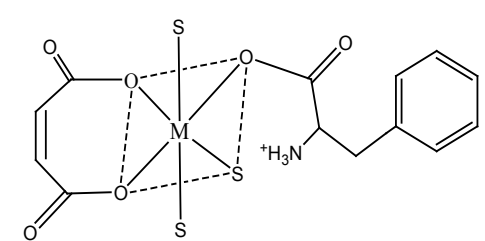

MLXH

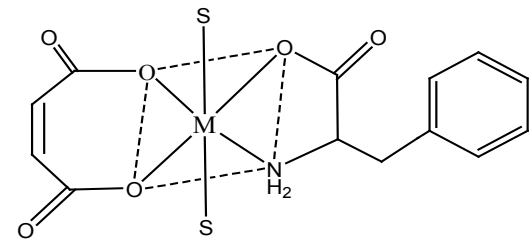

MLX

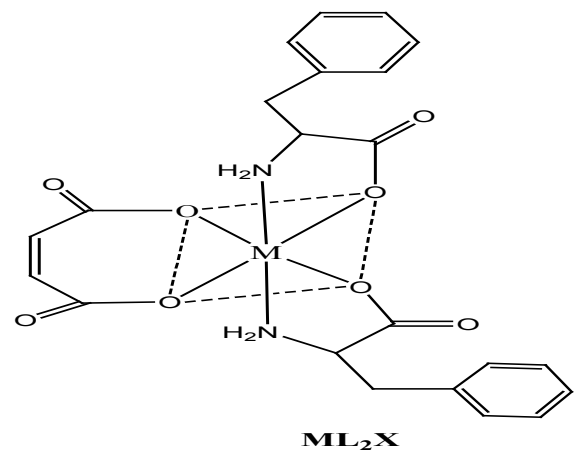

Figure 3. Proposed structures of ternary complexes of Fand MA with Co (II), Ni (II) and $\mathrm{Cu}$ (II), where Sis either solvent or water molecule. 
7) $\mathrm{ML}_{2} \mathrm{X}$ is expected antidote for $\mathrm{Cu}$ (II) around the biological (physiological) $\mathrm{PH}=7$. Therefore, it recommended justifying the structure of $\mathrm{ML}_{2} \mathrm{X}$.

\section{Conflicts of Interest}

The author declares no conflicts of interest regarding the publication of this paper.

\section{References}

[1] Nelson, D.L. and Cox, M.M., (2000) Lehninger, Principles of Biochemistry. 3rd Edition, Worth Publishing, New York.

[2] Koley, D. and Bard, A.J. (2010) Triton X-100 Concentration Effects on Membrane Permeability of a Single HeLa Cell by Scanning Electrochemical Microscopy (SECM). Proceedings of the National Academy of Sciences of the United States of America, 107, 16783-16787. https://doi.org/10.1073/pnas.1011614107

[3] Gordon, J.A. and Warren, J.R. (1968) Denaturation of Globular Proteins I. The Interaction of Urea and Thiourea with Bovine Plasma Albumin. Journal of Biological Chemistry, 243, 5663-5669.

[4] Hammes, G.G. and Swann, J.C. (1967) Influence of Denaturing Agents on Solvent Structure. Biochemistry, 6, 1591-1596. https://doi.org/10.1021/bi00858a004

[5] Wetiaufer, D.B., Malic, S.K., Stoller, L. and Coffin, R.L. (1964) Nonpolar Group Participation in the Denaturation of Proteins by Urea and Guanidinium Salts. Model Compound Studies. Journal of the American Chemical Society, 86, 508-514. https://doi.org/10.1021/ja01057a045

[6] Kresheck, G.C. and Benjamin, L. (1964) Calorimetric Studies of the Hydrophobic Nature of Several Protein Constituents and Ovalbumin in Water and in Aqueous Urea. The Journal of Physical Chemistry, 68, 2476-2486. https://doi.org/10.1021/j100791a015

[7] Schick, M.J. (1964) Effect of Electrolyte and Urea on Micelle Formation. The Journal of Physical Chemistry, 68, 3585-3592. https://doi.org/10.1021/j100794a025

[8] Waugh, D.F. (1954) Protein-Protein Interactions. Advances in Protein Chemistry, 9, 325-437. https://doi.org/10.1016/S0065-3233(08)60210-7

[9] Nageswara, R.C., Ramanaiah, M.M.M. and Sailaja, B.B.V. (2014) Influence of Dielectric Constant on Protonation Equilibria of Maleic Acid and L-Asparagine in Acetonitrile Water-Mixtures. Chemical Speciation \& Bioavailability, 26, 266-272. https://doi.org/10.3184/095422914X14037891051393

[10] Rao, G.N. and Ramakrishna, A. (2005) Speciation Studies of Nickel (II) Complexes of L-Glutamine and Succinic Acid in Urea-Water Mixtures. Proceedings of the National Academy of Sciences, India, 75, 245-248.

[11] Gran, G. (1988) Equivalence Volumes in Potentiometric Titrations. Analytica Chimica Acta, 206, 111-123. https://doi.org/10.1016/S0003-2670(00)80835-1

[12] Bates, R.G. and Dinching, G.D. (1951) Acidic Dissociation Constant and Related Thermodynamic Quantities for Monoethanolammonium Ion in Water from $0^{\circ}$ to $50^{\circ} \mathrm{C}$. Journal of Research of the National Bureau of Standards, 46, 349-352. https://doi.org/10.6028/jres.046.039

[13] Latha, M.P., Rao, V.M., Rao, T.S. and Rao, G.N. (2007) Chemical Speciation of Pb (II), Cd (II), Hg (II), Co (II), Ni (II), Cu (II) and Zn (II) Binary Complexes of 1-Methionine in 1, 2-Propanediol-Water Mixtures. Bulletin of the Chemical Society 
of Ethiopia, 21, 363-372.

[14] Gans, P., Sabatini, A. and Vacca, A. (1976) An Improved Computer Program for the Computation of Formation Constants from Potentiometric Data. Inorganica Chimica Acta, 18, 237-239. https://doi.org/10.1016/S0020-1693(00)95610-X

[15] Latha, M.P., Rao, V.M., Rao, T.S. and Rao, G.N. (2007) Determination of Protonation Constants of L-Glutamic Acid and L-Methionine in 1, 2-Propanediol-Water Mixtures. Acta Chimica Slovenica, 54, 160-165.

[16] Ramanaiah, M., Goutham Sri, S. and Sailaja, B.B.V. (2014) Chemical Speciation of PbII, Cd-II and Hg-II Binary Complexes of L-Phenylalanine in CTAB-Water Mixtures. Journal of the Indian Chemical Society, 91, 351-357.

[17] Ramanaiah, M. and Sailaja, B.B.V. (2014) pH-Metric Investigation on Binary Complexes of $\mathrm{Pb}$-II, Cd-II and Hg-II with Maleic Acid in SLS-Water Mixtures. Journal of the Indian Chemical Society, 91, 639-645.

[18] Rao, R.S. and Rao, G.N. (2005) Computer Applications in Chemistry. Himalaya Publishing House, Mumbai, India, 277-352.

[19] Hamilton, W.C. (1965) Significance Tests on the Crystallographic $R$ Factor. Acta Crystallographica, 18, 502-510. https://doi.org/10.1107/S0365110X65001081

[20] Rao, G.N. and Rao, R.S. (2005) Computer Application in Chemistry. Himalaya Publishing House, Mumbai, 277-351.

[21] Greisser, R. and Sigel, H. (1970) Ternary Complexes in Solution. VIII. Complex Formation between the Copper(II)-2,2'-Bipyridyl 1:1 Complex and Ligands Containing Oxygen and/or Nitrogen Donor Atoms. Inorganic Chemistry, 9, 1238-1243. https://doi.org/10.1021/ic50087a045

[22] Griesser, R. and Sigel, H. (1971) Ternary Complexes in Solution. XI. Complex Formation between the Cobalt(II)-, Nickel(II)-, Copper(II)-, and Zinc(II)-2,2'-Bipyridyl 1:1 Complexes and Ethylenediamine, Glycinate, or Pyrocatecholate. Inorganic Chemistry, 10, 2229-2232. https://doi.org/10.1021/ic50104a028

[23] Griesser, R. and Sigel, H. (1974) Ternary Complexes in Solution. XVI. Influence of the Size of the Chelate Rings on the Stability of Mixed-Ligand Copper(II) Complexes Containing Aliphatic Ligands. Inorganic Chemistry, 13, 462-465.

https://doi.org/10.1021/ic50132a046

[24] Sigel, H., Huber, P.R., Greisser, R. and Prijs, B. (1974) Ternary Complexes in Solution. XV. Mixed-Ligand Copper(II) Complexes with 2,2'-Bipyridyl or 1,10-Phenanthroline and Pyrocatecholate or Derivatives thereof. Inorganic Chemistry, 12, 11981200. https://doi.org/10.1021/ic50123a047

[25] Kida, S. (1956) Investigation on Mixed Complex. I. Spectrophotometric Study of Mixed Complexes Formed by Cupric Ion and Bidentate Ligands. Bulletin of the Chemical Society of Japan, 29, 805. https://doi.org/10.1246/bcsj.29.805

[26] Martin, R.B. and Prados, R. (1974) Some Factors Influencing Mixed Complex Formation. Journal of Inorganic and Nuclear Chemistry, 36, 1665-1670. https://doi.org/10.1016/0022-1902(74)80643-3

[27] Sigel, H., Becker, K. and McCormick, D.B. (1967) Ternary Complexes in Solution. Influence of 2,2'-Bipyridyl on the Stability of 1:1 Complexes of $\mathrm{Co}^{2+}, \mathrm{Ni}^{2+}, \mathrm{Cu}^{2+}$, and $\mathrm{Zn}^{2+}$ with Hydrogen Phosphate, Adenosine 5'-Monophosphate, and Adenosine 5'Triphosphate. Biochimica et Biophysica Acta, 148, 655-664.

[28] Sigel, H. (1975) Ternary $\mathrm{Cu}^{2+}$ Complexes: Stability, Structure, and Reactivity. Angewandte Chemie International Edition in English, 14, 394-402.

https://doi.org/10.1002/anie.197503941 
[29] Sakurai, T., Yamauchi, O. and Nakahara, A. (1977) Stereoselectivity in Mixed Ligand Copper(II) Complexes with Electrostatic Ligand-Ligand Interactions. Application to Optical Resolution of $\alpha$-Amino Acids with a Charged Side Chain. Bulletin of the Chemical Society of Japan, 50, 1776. https://doi.org/10.1246/bcsj.50.1776

[30] Nemethy, G. and Sheraga, H.A. (1962) Structure of Water and Hydrophobic Bonding in Proteins. II. Model for the Thermodynamic Properties of Aqueous Solutions of Hydrocarbons. The Journal of Chemical Physics, 36, 3401. https://doi.org/10.1063/1.1732473

[31] Singh, A.K. and Manjula, D. (2001) A Fluorescence Study of 1-p-Aminophenyl-4Phenylbuta-1E,3E-Diene in Organic Solvents, 1,4-Dioxane-Water Binary Mixtures and Micelles. Journal of the Indian Chemical Society, 78, 635-641.

[32] Cordes, E.H. (1978) Kinetics of Organic Reactions in Micelles. Pure and Applied Chemistry, 50, 617. https://doi.org/10.1351/pac197850070617 